\title{
Viscoelastic focusing of polydisperse particle suspensions in a straight circular microchannel
}

\author{
Francesco Del Giudice ${ }^{1}$ (1)
}

Received: 20 February 2019 / Accepted: 17 June 2019 / Published online: 27 June 2019

(c) The Author(s) 2019

\begin{abstract}
Flow cytometry is a technique for the analysis of cells and particles by coupling a sheath flow assisted focusing microfluidic device to an optical or electrical reader. In this context, polymer solutions can be employed to drive particle and cell focusing on the centreline of simple straight microfluidic channels. However, the change of the focusing efficiency due to polydispersity, which is a rule rather than the exception across cell populations, has not been extensively studied yet. In this work, the effect of particle polydispersity on the viscoelastic focusing in a straight cylindrical microchannel was studied by preparing two particle suspensions, containing different concentrations of particles with average diameters of $10 \mu \mathrm{m}, 15 \mu \mathrm{m}$ and $20 \mu \mathrm{m}$, suspended in three solutions of polyethylene oxide with mass concentrations of $0.1 \mathrm{wt} \%, 0.25 \mathrm{wt} \%$ and $0.5 \mathrm{wt} \%$. When the fluid explored the constant-viscosity region of the rheological curve, up to $95 \%$ of the particles were aligned on the channel centreline. When the fluid explored the shear-thinning region of the rheological curve, centreline focusing efficiency decreased, with the highest value of aligned particles being of $60 \%$. For both mixtures, it was also observed that the fraction of aligned particles in a polydisperse system was not equivalent to that derived from the estimate of independent experiments with monodisperse particles.
\end{abstract}

Keywords Microfluidic $\cdot$ Viscoelastic $\cdot$ Polydisperse $\cdot 3 \mathrm{D}$ focusing $\cdot$ Alignment

\section{Introduction}

Flow cytometry is a technique for the analysis of cells and particles, by direct interrogation with an optical or electrical source (Erickson and Li 2004). Despite being around since the 1960s, flow cytometry devices have been adopted for clinical analysis only in the last 30 years (Brown and Wittwer 2000). Indeed, technological advancements transformed a substantially bulky instrument into a more compact

This article is part of the topical collection "Particle motion in non-Newtonian microfluidics" guest edited by Xiangchun Xuan and Gaetano D'Avino.

Electronic supplementary material The online version of this article (https://doi.org/10.1007/s10404-019-2263-5) contains supplementary material, which is available to authorized users.

Francesco Del Giudice

francesco.delgiudice@swansea.ac.uk

1 Systems and Process Engineering Centre, College of Engineering, Swansea University, Fabian Way, Swansea SA1 8EN, UK device for fast clinical cytometry analysis. In the commercial cytometer, a suspension of flowing cells or particles is surrounded by two sheath flows which enable the focusing of the cells or the particles along the centreline of a microfluidic channel, achieving the so-called 3D focusing. Once focused, a laser source is used to derive clinical information on the cells suspended in the sample (Yin and Marshall 2012). To achieve 3D focusing in microfluidic devices, however, several sheath flows or more complicated channel designs are required, which eventually increases the complexity and costs of the system. For this reason, new techniques for manipulating the trajectories of particle and cells in microfluidic devices have been developed. Among the plethora of methodologies available, the use of nonlinear flow-induced forces for particle or cell manipulation recently gained much interest (Stoecklein and Di Carlo 2018). In these conditions, the trajectories of suspended particles are controlled by tuning the intensity of the inertial or elastic force (both nonlinear forces), or by controlling the relative magnitude of the two. In inertial focusing, particle and cells positions can be controlled by changing the flow rate and the geometry of the channel. However, particles and cells 
flowing in simple straight channels in inertial flow conditions occupy multiple equilibrium positions, which is not optimal for flow cytometry applications, where a single line of particles is desired to avoid cell or particle overlapping. To solve this problem, microfluidic devices with complex geometries have been introduced. For instance, Kim et al. (2016) designed a microfluidic device made of a series of straight microchannels with triangular and semi-circular cross-sections, to align and order particles on a single line. More recently, Zhang et al. (2018) presented an inertial microfluidic device made of several curvatures to achieve single-line focusing.

A novel methodology to 'tune' particle trajectories in microchannels by adding small quantities of polymers to the suspending liquid was introduced for the first time by Leshansky et al. (2007). The authors demonstrated that particles with a diameter of $8 \mu \mathrm{m}$ flowing in a shallow microchannel migrated transversally to the flow direction due to the elastic forces generated by the deformation of the polymer chains in the solution, as previously predicted by Ho and Leal (1976). At $2 \mathrm{~cm}$ from the inlet, particles were distributed on the middle plane of the shallow microchannel, occupying distinct focusing positions. Since their pioneering article, a considerable amount of work has been carried out to explore the potential of the viscoelastic fluids for particle and cell manipulation (see the detailed reviews of D'Avino et al. 2017; Lu et al. 2017; Yuan et al. 2018). The main advantage of this approach over other techniques for particle manipulation is that it is possible to achieve 3D single-line focusing on the centreline of a straight microchannel in sheathless conditions, which is very important to realise a viscoelasticity-based flow cytometer. Single-line particle focusing in a viscoelastic solution was demonstrated for microchannel with rectangular (Yang et al. 2011; Del Giudice et al. 2013; Lim et al. 2014; Holzner et al. 2017), as well as cylindrical cross-sections (D'Avino et al. 2012; Seo et al. 2014; Kang et al. 2013). Very recently, the first experimental evidence of particle ordering in viscoelastic liquids was also reported by Del Giudice et al. (2018), confirming the preliminary simulation carried out by D'Avino et al. (2013). Sorting of particles and cells with different dimensions and shapes has also been reported in simple straight microfluidic channels (Nam et al. 2012; Ahn et al. 2015; Liu et al. 2015; Lu and Xuan 2015; Li et al. 2016; D'Avino et al. 2019). The addition of channel curvature was shown to add further degrees of freedom in the manipulation of particle trajectories (Lee et al. 2013; Xiang et al. 2016; Liu et al. 2016). Viscoelastic liquids have also been used to expand the potential of well-established techniques. For instance, D'Avino (2013) and Li et al. (2018) showed numerically and experimentally that viscoelastic liquids can improve the efficiency of the well-established deterministic lateral displacement technology introduced by Huang et al.
(2004). Moreover, viscoelastic forces can be actively used for the manipulation of small Brownian particles (De Santo et al. 2014; Kim et al. 2012; Schlenk et al. 2018), in contrast with inertial forces that drop in magnitude when the diameter of particles decreases. Viscoelastic liquids have also been used for biomedical applications such as exosome separation (Liu et al. 2017), solution exchange (Yuan et al. 2016) and cell stretching (Cha et al. 2012). In this respect, there are only few works that presented a viscoelastic flow cytometer. Dannhauser et al. (2015) coupled the viscoelastic focusing in straight microchannel to a dynamic light scattering apparatus to analyse flowing cells. In their analysis, however, they did not discuss in details how the focusing efficiency was affected by cell polydispersity, which is the rule rather than the exception across cell populations (Yin and Marshall 2012). Very recently, Asghari et al. (2017) presented a viscoelastic flow cytometer for the analysis of $6 \mu \mathrm{m}$ particles, but they did not account for polydispersity. This limitation is important and can be difficult to overcome, as the magnitude of the viscoelastic force acting on particles or cells strongly depends on their size. To the best of our knowledge, however, there are no systematic studies on the effect of polydispersity on the centreline viscoelastic focusing of suspended particles or cells.

In this work, the effect of particle polydispersity on the viscoelastic focusing in a straight cylindrical microchannel was studied by preparing two particle suspensions labelled as MIX1 and MIX2, containing different concentrations of particles with diameters of $10 \pm 1 \mu \mathrm{m}, 15 \pm 1.5 \mu \mathrm{m}$ and $20 \pm 2 \mu \mathrm{m}$, suspended in three solutions of polyethylene oxide (PEO) with mass concentrations of $0.1 \mathrm{wt} \%, 0.25 \mathrm{wt} \%$ and $0.5 \mathrm{wt} \%$. When the fluid explored the constant-viscosity region of the rheological curve, up to $95 \%$ of the particles were aligned on the channel centreline. When the fluid explored the shear-thinning region of the rheological curve, centreline focusing efficiency decreased, with the highest value of aligned particles being of $60 \%$. For both mixtures, it was also observed that the fraction of aligned particles in a polydisperse system was not equivalent to that derived from the estimate of independent experiments with monodisperse particles. This phenomenon was ascribed to potential hydrodynamic interactions between particles with different diameters in confined flows, even though the suspension was macroscopically considered dilute.

\section{Materials and methods}

\subsection{Preparation and characterisation of the suspending fluids}

Polyethylene oxide (PEO) with average molecular weight $M_{\mathrm{w}}=4 \mathrm{MDa}$ (Sigma Aldrich, UK) was dispersed in water 
at the three mass concentrations of $0.1 \mathrm{wt} \%, 0.25 \mathrm{wt} \%$, and $0.5 \mathrm{wt} \%$. Glycerol (Sigma Aldrich, UK) at $25 \mathrm{wt} \%$ was added to the aqueous solutions to prevent particle sedimentation. The viscosity of the glycerol-water solution was $\eta_{\mathrm{s}}=2 \times 10^{-3} \mathrm{Pas}$.

The viscosity curves of the three solutions were evaluated through a stress-controlled TA AR2000ex rheometer, at $T=25^{\circ} \mathrm{C}$ (Fig. 1a). An acrylic cone with $60 \mathrm{~mm}$ diameter was used together with a home made solvent-trap to avoid fluid evaporation. The PEO $0.1 \mathrm{wt} \%$ displayed a near constant-viscosity in the whole range of shear rates investigated (Fig. 1a). PEO $0.25 \mathrm{wt} \%$ and PEO $0.5 \mathrm{wt} \%$ presented a constant-viscosity region at low shear rates, and then displayed shear-thinning features above a critical value of shear rate $\dot{\gamma}_{\mathrm{c}}$ (Fig. 1a). The degree of shear-thinning for PEO $0.25 \mathrm{wt} \%$ and PEO $0.5 \mathrm{wt} \%$ was quantified by fitting the high shear rate region of the viscosity curve with the power law model (Bird et al. 1987), i.e., $\eta=m \dot{\gamma}^{n-1}$, where $n=0.784$ and $n=0.618$ are the best fit values of the flow-index for PEO $0.25 \mathrm{wt} \%$ and for PEO $0.5 \mathrm{wt} \%$, respectively, and $m$ is a prefactor.

The longest relaxation time values for the three fluids were evaluated using a $\mu$-rheometer introduced previously by Del Giudice et al. (2015a), based on the viscoelastic transversal migration of particles in straight microchannels. For this measurement, a glass capillary with internal diameter $D=150 \mu \mathrm{m}$ and length $L=6.5 \mathrm{~cm}$ was employed. The fraction of $15 \mu \mathrm{m}$ particles suspended in the three fluids (volumetric concentration of $\phi=0.1 \%$ ) that aligned on the channel centreline was evaluated. The obtained longest relaxation time values were $\lambda=0.02 \mathrm{~s}$ for PEO $0.1 \mathrm{wt} \%, \lambda=0.034 \mathrm{~s}$ for PEO $0.25 \mathrm{wt} \%$ and $\lambda=0.2 \mathrm{~s}$ for PEO $0.5 \mathrm{wt} \%$. The interested reader may refer to the original paper by Del Giudice et al. (2015a) for the working principle details.

\subsection{Preparation of the particle suspensions}

To test the effect of polydispersity on the viscoelastic alignment, two suspensions of polystyrene particles (Polysciences
Inc.), called MIX 1 and MIX 2, having each a total volumetric concentration of $\phi=0.1 \mathrm{vol} \%$, were prepared. In each mixture, particles with three diameters $d_{\mathrm{p}}=10 \pm 1 \mu \mathrm{m}$, $d_{\mathrm{p}}=15 \pm 1.5 \mu \mathrm{m}$, and $d_{\mathrm{p}}=20 \pm 2 \mu \mathrm{m}$ were added. The volume fractions of each set of particles in the two suspensions were $x_{10}=0.275 \phi, x_{15}=0.45 \phi, x_{20}=0.275 \phi$ for MIX1; while they were $x_{10}=0.31 \phi, x_{15}=0.25 \phi, x_{20}=0.34 \phi$ for MIX2.

\subsection{Experimental methodology}

An inverted microscope (Zeiss Axiovert 135) in bright field with a $5 \times$ objective (Zeiss Objective Epiplan $5 \times / 0.13$ W0.8, $\mathrm{WD}=20.5 \mathrm{~mm}$ ) was employed to observe the particles flowing in the circular glass microcapillaries with diameter $D=150 \mu \mathrm{m}$. In analogy with previous works (Del Giudice et al. 2013; Yang et al. 2011), particle positions were monitored at a fixed distance $L_{\mathrm{z}}=55 \mathrm{~mm}$ from the channel inlet (Fig. 1b). The capillary was glued to a 250 -mm-long silicone tube with internal diameter of $250 \mu \mathrm{m}$ and external diameter of $1.6 \mathrm{~mm}$. Silicone tubes were connected to a pressure pump (Mitos p-pump, Dolomite microfluidics), operating in the range of pressure drops $30<\Delta p<480$ mbar. Images of flowing particles were recorded using a fast camera (Photron, fastcam Mini UX50) at a frame rate variable between 50 and $8000 \mathrm{fps}$ depending on the imposed pressure drop. All the experiments were performed at $T=25^{\circ} \mathrm{C}$.

In agreement with the previous work by De Santo et al. (2014), the circular channel cross-section was divided into 5 bands $k$, each of radius $\Delta r=15 \mu \mathrm{m}$. The normalised fraction of particles in each band $k$ was then evaluated according to the definition presented by D'Avino et al. (2012):

$f_{k}\left(L_{z}\right)=\frac{\frac{n_{k}\left(L_{z}\right)}{A_{k} \bar{v}_{k}}}{\sum_{k} \frac{n_{k}\left(L_{z}\right)}{A_{k} \bar{v}_{k}}}$,

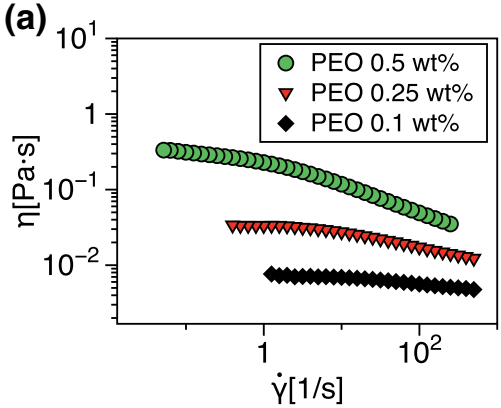

Fig. 1 a Shear viscosity $\eta$ as a function of the shear rate $\dot{\gamma}$ for PEO $0.1 \mathrm{wt} \%$ (black diamonds), PEO $0.25 \mathrm{wt} \%$ (red triangles) and PEO 0.5 wt\% (green circles). b Schematic of the experimental setup (b)

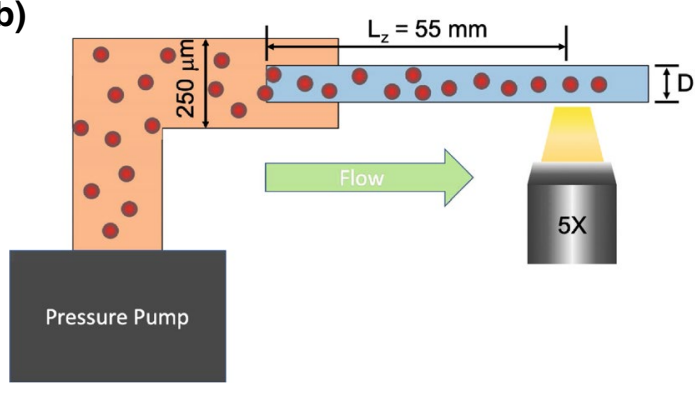

employed for the experimental campaign. Green rectangle is the glass capillary, and orange rectangle is the silicone connecting tube. The channel diameter is $D=150 \mu \mathrm{m}$. Dimensions are not in scale 
where $n_{k}\left(L_{z}\right)$ is the number of particles flowing in the band $k$, and $A_{k}$ and $\bar{v}_{k}$ are the cross-sectional area and the average velocity of the band $k$ (fluid without particles), respectively. Hereafter, the fraction of aligned particles will be indicated with the symbol $f_{1}$.

The number $n_{k}$ of particles belonging to each band $k$ was derived by employing a relation between the radial coordinate $r$ and the particle velocity in that point $V_{z}(r)$. For power law-fluids with flow-index $n$, the relation between radial position and velocity is written as (Bird et al. 1987):

$\frac{r}{R}=\left(1-\frac{V_{z}(r)}{V_{z, \max }}\right)^{\frac{n}{n+1}}$,

where $V_{z, \max }$ is the maximum translational velocity evaluated from the particle tracking, and $n$ is the flow-index of the fluid derived from the rheology curves (Fig. 1a). For the correct application of Eq. (2) to the experimental results, several aspects need to be discussed in details. First, it is assumed that the particle velocity is equal to the fluid velocity. For confinement ratios $\beta=d / D$ employed here, the error committed by approximating the particle velocity as that of the fluid was less than $0.6 \%$ around the centreline and $2-3 \%$ near the walls, as reported by Higdon and Muldowney (1995). Therefore, the velocity of the particles could be approximated to that of the fluid. The numerical expression of Eq. (2) depends on the imposed flow rates (or pressure drops), as PEO $0.1 \mathrm{wt} \%$ is a near constant-viscosity fluid, while PEO $0.25 \mathrm{wt} \%$ and PEO $0.5 \mathrm{wt} \%$ both display a constant-viscosity region followed by a shear-thinning region (quantified by the flow-index $n$ ) at high shear rate values. The critical shear rate above which the fluids display shear-thinning features was evaluated by fitting the viscosity data with the Bird-Carreau model (Bird and Carreau 1968, curves not shown), being $\dot{\gamma}_{\mathrm{c}}=4.67 \mathrm{~s}^{-1}$ for PEO $0.25 \mathrm{wt} \%$ and $\dot{\gamma}_{\mathrm{c}}=1.2 \mathrm{~s}^{-1}$ for PEO $0.5 \mathrm{wt} \%$. By defining the average shear rate in the microfluidic channel as $\bar{\gamma}=4 Q /\left(\pi D^{3}\right)$, the value of the critical flow rates $Q_{\mathrm{c}}$ above which the velocity profile in the channel changed from parabolic to pseudoparabolic was obtained (black dotted lines of Fig. 2b, c). The relation between the flow rate and the pressure drop in a straight circular tube can be written as (Bird et al. 1987):

$\Delta p=\mathcal{R} Q^{n}$,

where the expression for $\mathcal{R}$ is:

$\mathcal{R}=2 m\left(\frac{\frac{1}{n}+3}{\pi R^{3}}\right)^{n} \frac{L}{R} Q^{n}$,

where $m$ and $n$ are the parameters derived from the fitting of the viscosity curves in the shear-thinning region with the power law model. The experimental setup employed here (a)
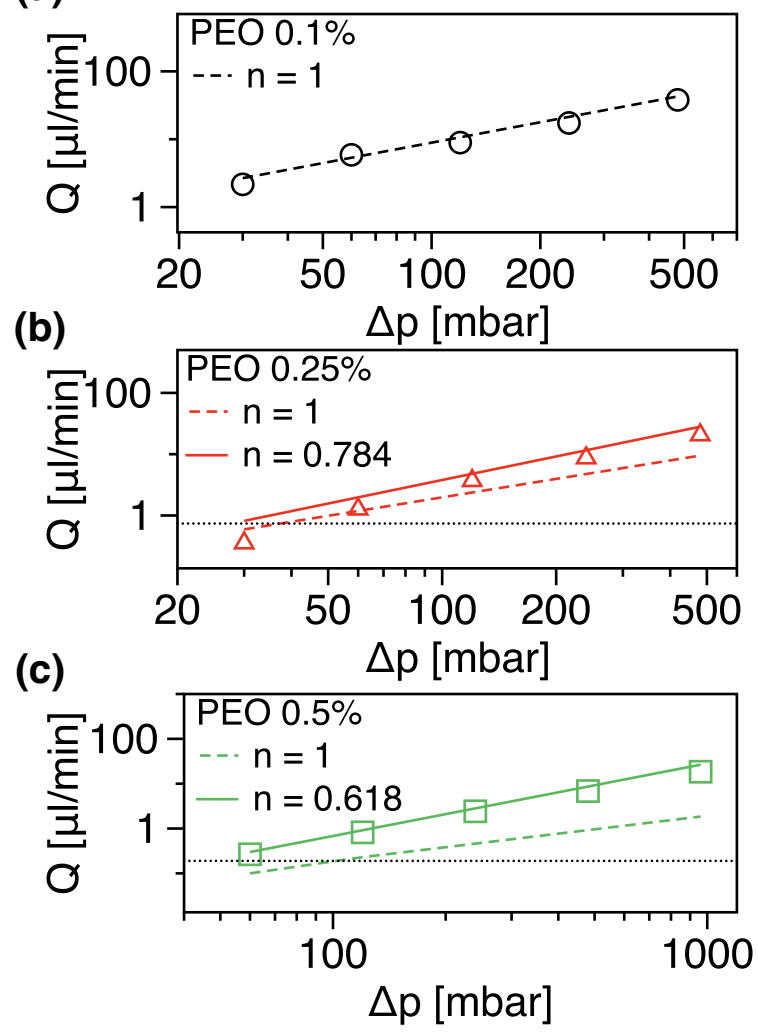

Fig. 2 Estimated volumetric flow rate $Q$ from the maximum velocity $V_{z, \max }$ obtained through particle tracking (see main text for more details) as a function of the imposed pressure drop $\Delta p$. Open circles are for PEO $0.1 \mathrm{wt} \%$ (a), red triangles are for PEO $0.25 \mathrm{wt} \%$ (b), and green squares are for PEO $0.5 \mathrm{wt} \%$ (c). Dashed and solid lines are Eq. (6) for $n=1$ and $n \neq 1$, respectively. Black dotted line in $\mathbf{b}, \mathbf{c}$ is the estimated value of the critical flow rate $Q_{\mathrm{c}}$ above which the velocity profile in the microchannel is no longer parabolic

was made of two capillaries in series (Fig. 1b), therefore, the total pressure drop was given by:

$\Delta p=\Delta p_{\mathrm{s}}+\Delta p_{\mathrm{g}}$,

where the subscripts $\mathrm{s}$ and $\mathrm{g}$ refer to silicone and glass, respectively. Since the flow rate is constant for two capillaries in series, it is possible to write:

$Q=\left(\frac{\Delta p}{\mathcal{R}_{\mathrm{s}}+\mathcal{R}_{\mathrm{g}}}\right)^{\frac{1}{n}}$

When the imposed flow rate $Q$ is smaller than the critical flow rate $Q_{\mathrm{c}}$, the relation between radial position and velocity is given by Eq. (2) with $n=1$. When the imposed flow rate exceeds the critical value, such relation is obtained by replacing in Eq. (2) the value of the flow-index $n$ for the fluid under investigation. For each fluid, good agreement between the values of the average flow rate derived from the maximum velocity of the particles at the centreline $V_{z, \max }$ and the 
theoretical values of flow rate evaluated through Eq. (6) was found (Fig. 2a-c).

Finally, it was verified that Eq. (2) could be used for a polydisperse system, as particle with different diameters may display different velocities even when they flow on the same streamline. From the recorded videos, the velocities of flowing particles with different diameters were manually measured. For particles suspended in PEO $0.1 \mathrm{wt} \%$, the discrepancy between their centreline velocity values was less than $1 \%$ at $\Delta p=30$ mbar while being $\sim 2 \%$ at $\Delta p=480$ mbar. For particles suspended in PEO $0.5 \mathrm{wt} \%$, the discrepancy between their centreline velocity values was found to be less than $1 \%$ at both $\Delta p=60$ mbar and $\Delta p=480$ mbar.

The obtained values of the flow rate were employed for the calculation of the Deborah number in the microcapillary for each imposed pressure drop, defined as $D e=\lambda \overline{\dot{\gamma}}$, where $\bar{\gamma}=4 Q /\left(\pi D^{3}\right)$ is the average shear rate in the circular microchannel (Table 1). In Table 1, we also report the values of the Reynolds number $R e=4 \rho Q /(\pi \eta D)$, and of the elasticity number $E l=D e / R e$. These dimensionless parameters are important to quantify the magnitude of inertial and viscoelastic forces.

\section{Results and discussion}

At relatively low Deborah numbers $D e$, the magnitude of the viscoelastic force acting on flowing particles scales with the square of the confinement ratio $\beta=d / D$, as reported

Table 1 Estimated values of Deborah number $D e=4 \lambda Q /\left(\pi D^{3}\right)$, Reynolds number $\operatorname{Re}=4 \rho Q /(\pi \eta D)$ and elasticity number $E l$ in the microcapillary, for each imposed pressure drop, for the three fluids PEO $0.1 \mathrm{wt} \%$, PEO $0.25 \mathrm{wt} \%$ and PEO $0.5 \mathrm{wt} \%$

\begin{tabular}{|c|c|c|c|c|}
\hline$\Delta p$ (mbar) & $Q(\mu 1 / \min )$ & $D e$ & $R e$ & $E l=D e / R e$ \\
\hline \multicolumn{5}{|c|}{ Polyethylene oxide (PEO) $0.1 \mathrm{wt} \%$} \\
\hline 30 & 2.10 & 0.26 & 0.029 & 8.9 \\
\hline 60 & 5.72 & 0.72 & 0.086 & 8.3 \\
\hline 120 & 8.67 & 1.1 & 0.13 & 8.02 \\
\hline 480 & 4.65 & 26.9 & 0.67 & 6.9 \\
\hline \multicolumn{5}{|c|}{ Polyethylene oxide (PEO) $0.25 w t \%$} \\
\hline 60 & 1.19 & 0.25 & $3.1 \times 10^{-3}$ & 83 \\
\hline 120 & 3.69 & 0.74 & 0.01 & 69 \\
\hline 240 & 8.68 & 1.85 & 0.032 & 57.3 \\
\hline 480 & 20.6 & 4.39 & 0.092 & 47.6 \\
\hline \multicolumn{5}{|c|}{ Polyethylene oxide (PEO) $0.5 w t \%$} \\
\hline 60 & 0.27 & 0.23 & $9.7 \times 10^{-5}$ & 2409 \\
\hline 120 & 0.82 & 0.72 & $4.1 \times 10^{-4}$ & 1758 \\
\hline 240 & 2.42 & 2.13 & $1.7 \times 10^{-3}$ & 1214 \\
\hline 480 & 6.85 & 6.03 & $7.4 \times 10^{-3}$ & 815 \\
\hline
\end{tabular}

The viscosity used in the dimensionless numbers has been evaluated at the average shear rate $\bar{\gamma}=4 Q /\left(\pi D^{3}\right)$ by D'Avino et al. (2012). Therefore, particle polydispersity may dramatically limit the efficiency and accuracy of the centreline focusing. The viscoelastic focusing of the particle mixture MIX 1 in PEO $0.1 \mathrm{wt} \%$ was first studied (Fig. 3a-d). At $\Delta p=30 \mathrm{mbar}(D e=0.26)$, slightly more than $70 \%$ of the particles were focused near the centreline of the channel, with the $10 \mu \mathrm{m}$ particles being slightly offcentred (Fig. 3a). By increasing the imposed pressure drop to $\Delta p=60 \mathrm{mbar}$, over $95 \%$ of the particles were focused around the centreline, regardless of their particle diameter (Fig. 3b, Video S1). By increasing further the imposed pressure drop up to $\Delta p=480 \mathrm{mbar}$ (Fig. $3 \mathrm{c}, \mathrm{d}$ ), however, a steady decrease in the centreline focusing efficiency was observed, with only $50 \%$ of all the particles focused on the centreline at $\Delta p=480 \mathrm{mbar}$ (Fig. 3d). By looking at the experimental snapshots and at Video S2, particles with diameter of $10 \mu \mathrm{m}$ were found to be more scattered compared to the bigger ones. To understand these results, it is first necessary to quantify the magnitude of inertial and elastic force for this system. As previously reported by Del Giudice et al. (2015b), the ratio between the magnitude of elastic force and inertial force acting on suspended particles can be expressed as:

$\left|\frac{V_{\mathrm{m}}^{\mathrm{el}}}{V_{\mathrm{m}}^{\mathrm{in}}}\right| \propto \frac{E l}{\beta}$,

where $\left|V_{\mathrm{m}}^{\mathrm{el}}\right|$ is the magnitude of the elastic transversal migration of particles, while $\left|V_{\mathrm{m}}^{\text {in }}\right|$ is the magnitude of the inertial transversal migration of particles. In the investigated system, even at the smallest elasticity number $E l=6.9$ and at the highest confinement ratio $\beta=d / D=20 / 150=0.13$, the velocity ratio was $\left|V_{\mathrm{m}}^{\mathrm{el}}\right| /\left|V_{\mathrm{m}}^{\mathrm{in}}\right| \simeq 53$, thus inertial effects are supposed to be negligible. As reported by D'Avino et al. (2012), the magnitude of elastic migration in purely viscoelastic flows at low Deborah numbers can be quantified as:

$\left|V_{\mathrm{m}}^{\mathrm{el}}\right| \propto D e \beta^{2} \frac{r}{R}$,

where $r$ is the radial coordinate and $R$ is the microchannel radius. An increase of the Deborah number $D e$ results in an increase of magnitude of the elastic migration, thus improving the migration towards the centreline, as also observed experimentally up to $D e=0.72(\Delta p=60 \mathrm{mbar})$. When increasing further the Deborah number at $D e>1$, Eq. (8) does not hold anymore due to the presence of shear-thinning features in the fluid. As previously experimentally observed by Seo et al. (2014) and Del Giudice et al. (2017), shearthinning is detrimental for particle alignment at values of $\beta \leq 0.1$, as particle start to migrate towards the corners of a straight square-shaped microchannel. For cylindrical microchannels and at $\beta \leq 0.1$, D'Avino et al. (2012) numerically predicted migration towards the lateral wall of a cylindrical 
(a)
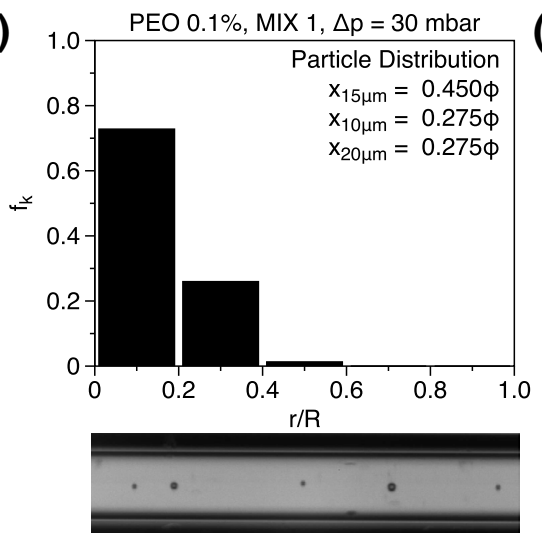

(d)
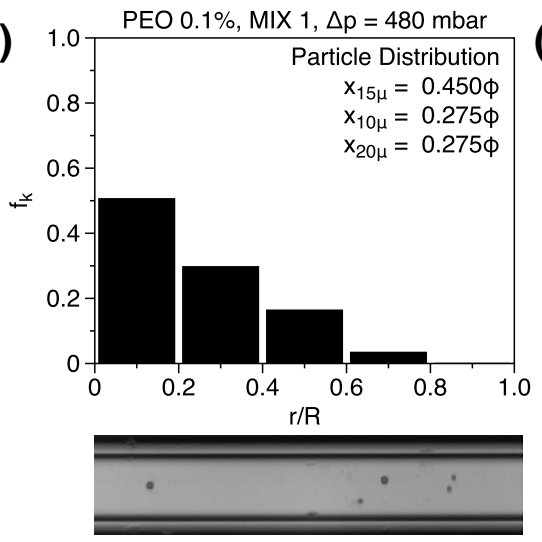

(g)
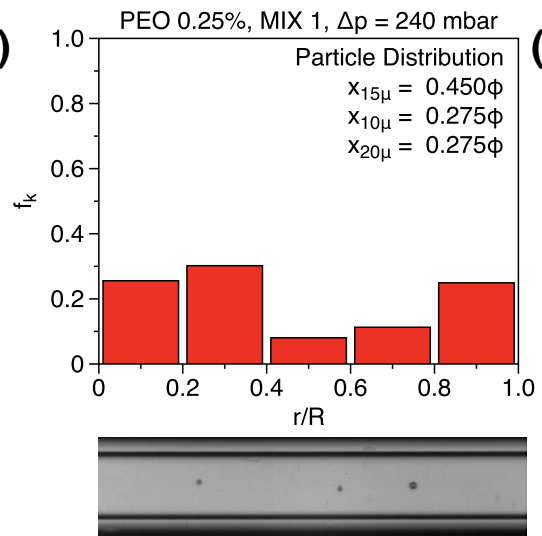

(I)
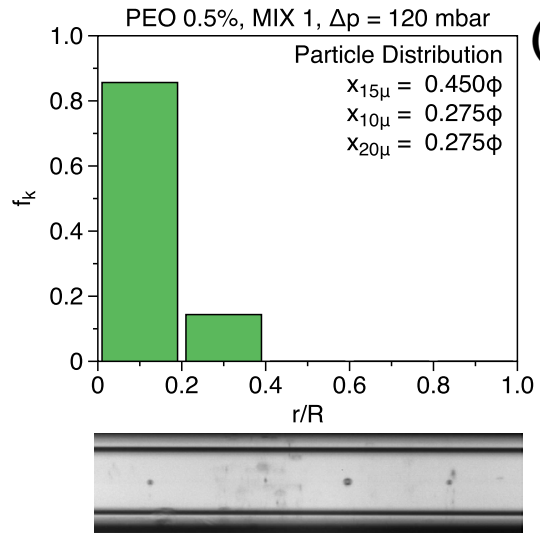

(b)
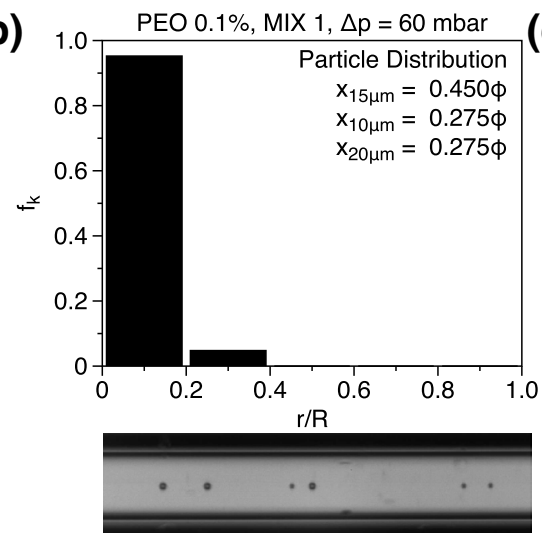

(e) 1.0 PEO $0.25 \%, M I X 1, \Delta p=60 \mathrm{mbar}$
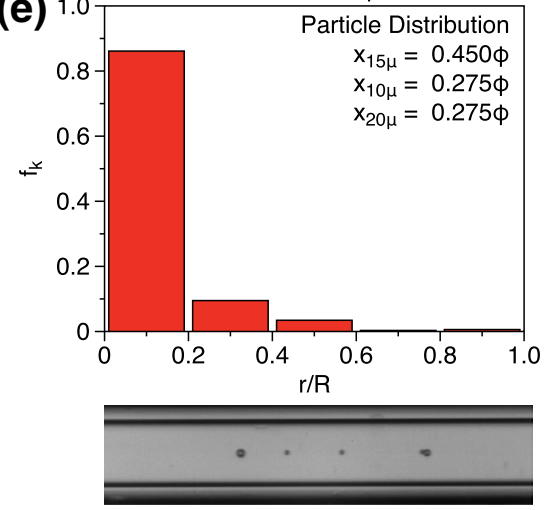

(h) 1.0 PEO 0.25\%, MIX 1, $\Delta p=480 \mathrm{mbar}$
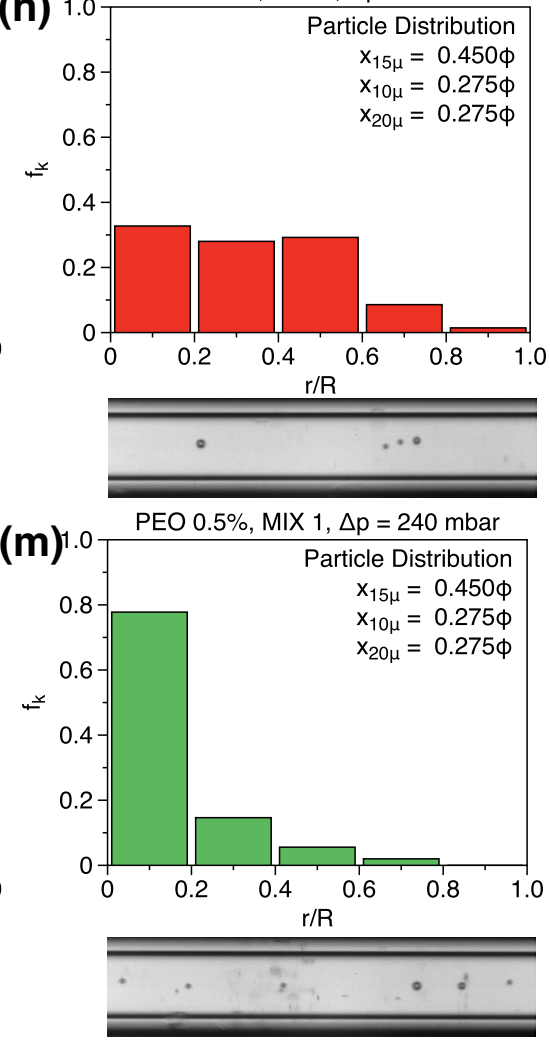

(C) 1.0 PEO 0.1\%, MIX 1, $\Delta p=120 \mathrm{mbar}$
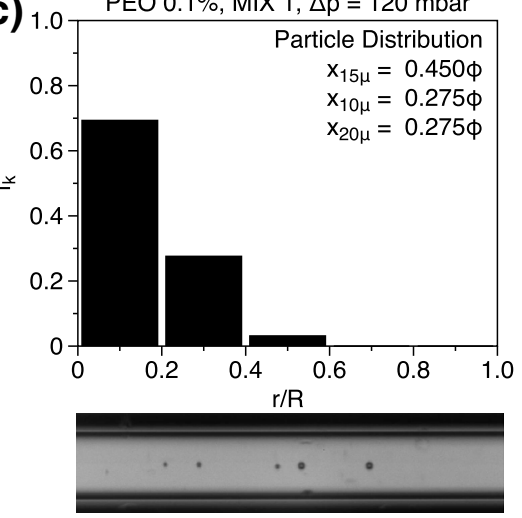

(f) 1.0 PEO $0.25 \%, M I X 1, \Delta p=120 \mathrm{mbar}$
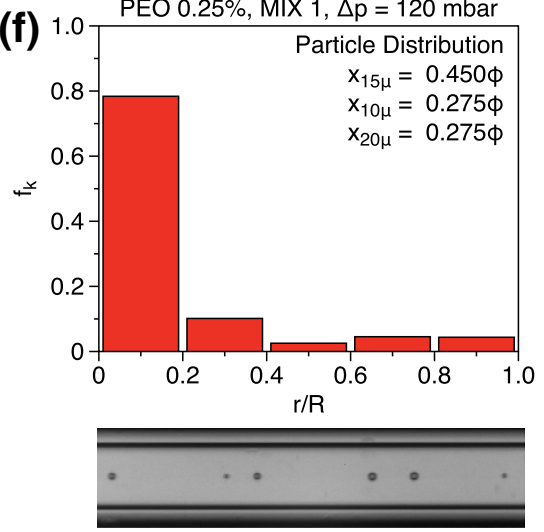

PEO $0.5 \%, M I X 1, \Delta p=60 \mathrm{mbar}$

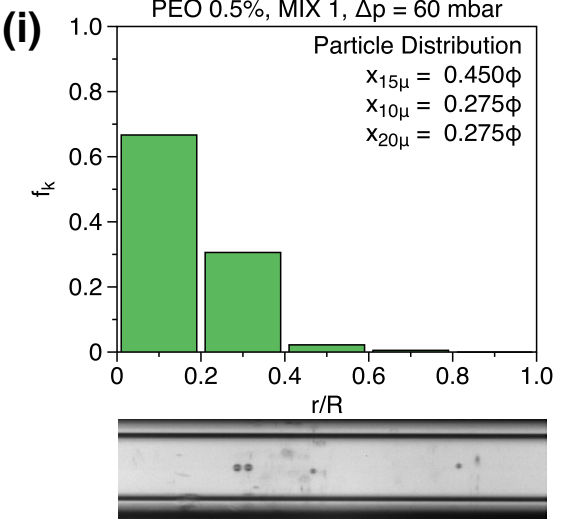

(n) 1.0 PEO 0.5\%,MIX 1, $\triangle \mathrm{p}=480 \mathrm{mbar}$

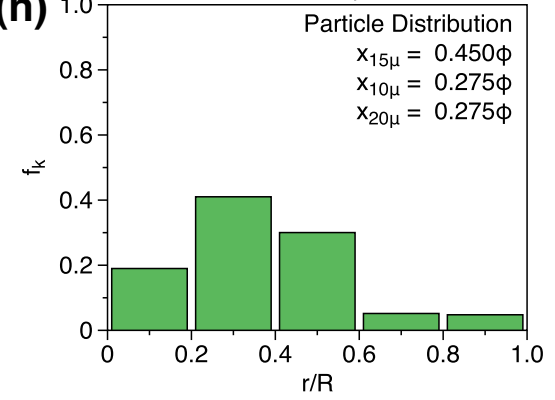


4Fig. 3 Normalised particle fraction $f_{k}$ (Eq. 1) as a function of the normalised radial position for the mixture MIX1. a-d Distribution for the PEO $0.1 \mathrm{wt} \%$ at different pressure drops $\Delta p$ in the range of $30<\Delta p<480$ mbar. Experimental snapshots are also reported below each graph. e-h Distribution for the PEO $0.25 \mathrm{wt} \%$ at different pressure drops $\Delta p$ in the range of $60<\Delta p<480$ mbar. i-n Distribution for the PEO $0.5 \mathrm{wt} \%$ at different pressure drops $\Delta p$ in the range of $60<\Delta p<480 \mathrm{mbar}$. Flow is from left to right. Channel internal diameter is $150 \mu \mathrm{m}$. The normalised fraction of particles $f_{k}$ was evaluated through the tracking of at least 100 particles

microchannel for particle suspended in a shear-thinning liquid. However, the shear-thinning of the PEO $0.1 \%$ is very mild (Fig. 1a) and it is more likely that the focusing equilibrium position remains the centreline (as observed by the histograms of Fig. 3), even though the magnitude of the elastic force which drives transversal migration towards the centreline decreases as a consequence of the shear-thinning. In other words, the presence of a mild shear-thinning may not be sufficient to promote migration towards the channel walls but it can rather lead to an increase of the channel length required to focus the particles; thus, longer channels are required to increase the fraction of particles focused on the centreline. This aspect is even more important in polydisperse systems, where the focusing length is actually dictated by smaller particles, as the migration velocity strongly depends on the particle diameter. These results are in good agreement with those presented by Song et al. (2016) on the transversal migration of monodisperse microparticles in rectangular microchannels. They observed that particles suspended in a mild shear-thinning PEO solution began to scatter around the centreline when $\eta / \eta_{0} \simeq 0.77$. In our case, the fraction of particles aligned on the centreline start to decrease at $\Delta p=120$ mbar, when $\eta / \eta_{0} \simeq 0.79$.

The experimental campaign was repeated for the same mixture of particles MIX1, suspended in the more elastic PEO $0.25 \mathrm{wt} \%$ (Fig. 3e-h). At $\Delta p=60 \mathrm{mbar}(D e=0.25)$, around $80 \%$ of particles were focused on the channel centreline, and they remained substantially aligned up to $\Delta p=120 \mathrm{mbar}$ $(D e=0.74)$. As for the PEO $0.1 \mathrm{wt} \%$, particles with diameter of $10 \mu \mathrm{m}$ were those off-centred (see experimental snapshots). These results are in-line with the previous findings on the focusing of polydisperse suspensions in PEO $0.1 \mathrm{wt} \%$, and can be explained using the same arguments. It is worth noticing, however, that at $D e \simeq 0.7$, the fraction of particles focused at the centreline was $f_{1} \sim 0.95$ for the PEO $0.1 \mathrm{wt} \%$ and $f_{1} \sim 0.8$ for PEO $0.25 \mathrm{wt} \%$. This discrepancy was ascribed to the different degree of shear-thinning for both fluids, being $n \simeq 1$ for PEO $0.1 \mathrm{wt} \%$ and $n=0.73$ for PEO $0.25 \mathrm{wt} \%$. In squareshaped microchannels, Del Giudice et al. (2015b) observed that for the more shear-thinning PEO $1.6 \mathrm{wt} \%$, the fraction of monodisperse $10 \mu \mathrm{m}$ particles focused on the centreline at $D e=0.3$ (i.e., in the constant-viscosity region) was significantly smaller than the fraction of focused particles observed at $D e=0.13$ for the PEO $0.8 \mathrm{wt} \%$ with more mild shear-thinning features. By increasing the imposed pressure drop further from $\Delta p=240 \operatorname{mbar}(D e=1.85)$ to $\Delta p=480 \operatorname{mbar}(D e=4.39)$, particles were not focused on the centreline anymore, but they were rather scattered over the whole cross-section (Fig. 3g, h). This is in agreement with the previous findings of D'Avino et al. (2012) and Seo et al. (2014) as, when $D e>1$, the fluid explores the shear-thinning region of the viscosity curve, thus the particle alignment is reduced.

To further test the effect of shear-thinning on the viscoelastic focusing of polydisperse particle suspensions, additional experiments were carried out on the mixture of particles MIX 1, suspended in the more elastic and shear-thinning PEO $0.5 \mathrm{wt} \%$ (Fig. 3i-n). At $\Delta p=60 \mathrm{mbar}(D e=0.23)$, around $67 \%$ of the particles were focused on the centreline, in good agreement with the results for PEO $0.1 \mathrm{wt} \%$ (Fig. 3a, Video S3), at the same value of the Deborah number De. By further increasing the imposed pressure drop, the fraction of aligned particles first reached the maximum value $f_{1}=0.86$ at $D e=0.72$ (in quantitative agreement with the findings for PEO $0.25 \mathrm{wt} \%$ at $D e=0.74$ reported in Fig. 3f), and then subsequently decreased until $\Delta p=480 \operatorname{mbar}(D e=6.03)$, where it was not possible to observe alignment (Fig. 3n, Video S4). The results at $D e>1$ (shear-thinning region) for PEO $0.25 \mathrm{wt} \%$ and for PEO $0.5 \mathrm{wt} \%$ further confirm that
Table 2 Comparison between the $f_{\text {aligned }}, f_{\text {poly }}$ and $f_{1}$

\begin{tabular}{|c|c|c|c|c|c|c|c|c|}
\hline$\Delta p$ (mbar) & $D e$ & $f_{\text {aligned, } 10}$ & $f_{\text {aligned,15 }}$ & $f_{\text {aligned,20 }}$ & $f_{\text {poly,MIX1 }}$ & $f_{1, \mathrm{MIX} 1}$ & $f_{\text {poly,MIX2 }}$ & $f_{2, \mathrm{MIX} 2}$ \\
\hline \multicolumn{9}{|c|}{ Polyethylene oxide (PEO) $0.1 w t \%$} \\
\hline 30 & 0.26 & 0.382 & 0.831 & 0.999 & 0.753 & 0.728 & 0.749 & 0.433 \\
\hline 60 & 0.71 & 0.942 & 0.9999 & 1 & 0.984 & 0.952 & 0.982 & 0.716 \\
\hline \multicolumn{9}{|c|}{ Polyethylene oxide (PEO) 0.25 wt\% } \\
\hline 60 & 0.25 & 0.373 & 0.801 & 0.998 & 0.737 & 0.861 & 0.735 & 0.809 \\
\hline 120 & 0.74 & 0.972 & 1 & 1 & 0.992 & 0.784 & 0.991 & 0.669 \\
\hline \multicolumn{9}{|c|}{ Polyethylene oxide (PEO) $0.5 \mathrm{wt} \%$} \\
\hline 60 & 0.23 & 0.356 & 0.739 & 0.995 & 0.704 & 0.667 & 0.707 & 0.351 \\
\hline 120 & 0.72 & 0.944 & 0.999 & 1 & 0.985 & 0.856 & 0.983 & 0.605 \\
\hline
\end{tabular}

Values of $f_{\text {aligned }}$ were derived through Eq. (9) for each particle diameter. Values of $f_{\text {poly }}$ were derived through Eq. (11), while $f_{1}$ was measured experimentally (Figs. 3, 4) 
(a)
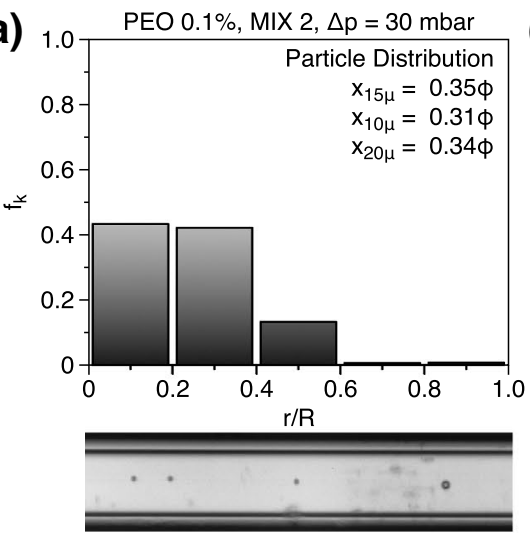

(d) 1.0 PEO $0.1 \%, M I X 2, \Delta p=480$ mbar
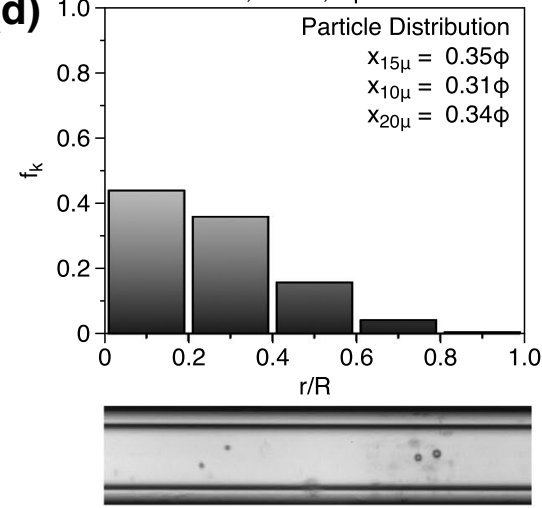

(g)
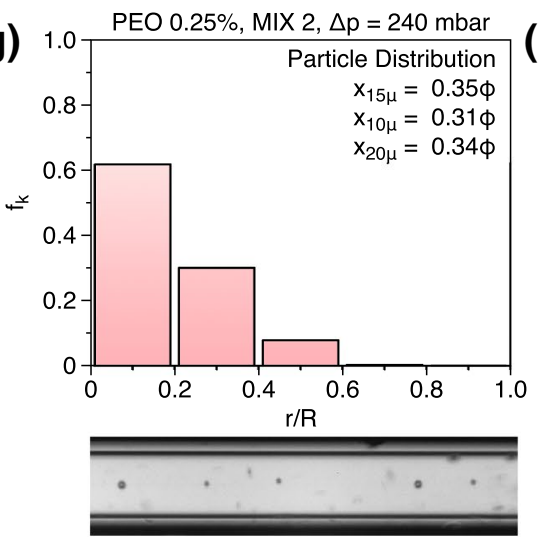

(I) 1.0 PEO 0.5\%, MIX 2, $\Delta p=120 \mathrm{mbar}$
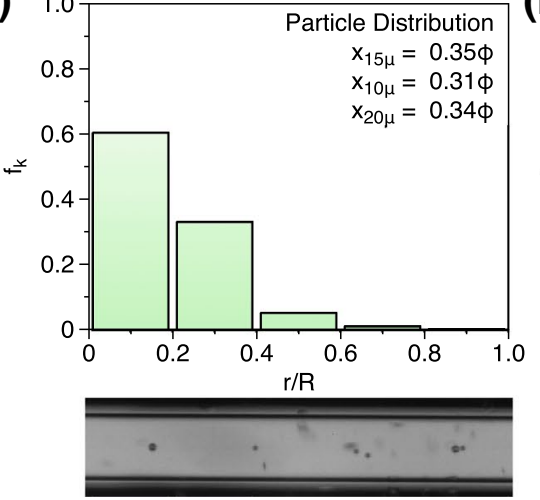

(b)
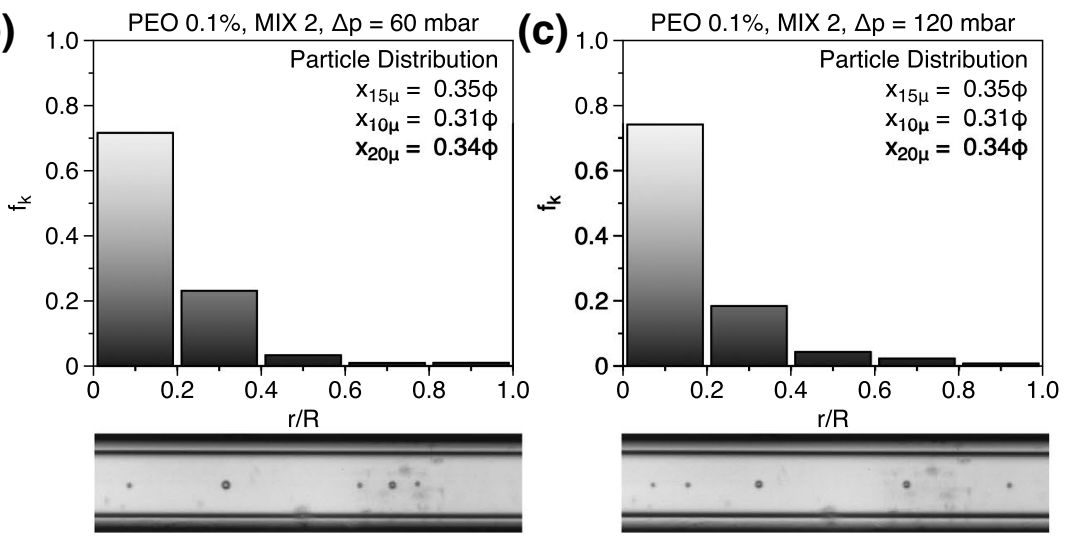

(e) 1.0 PEO $0.25 \%, M I X 2, \Delta p=60 \mathrm{mbar}$

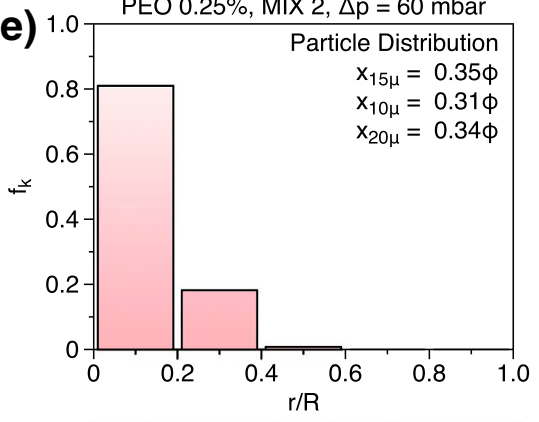

(f)

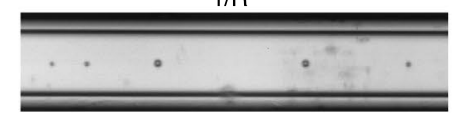

PEO $0.25 \%, \operatorname{MIX} 2, \Delta p=120$ mbar
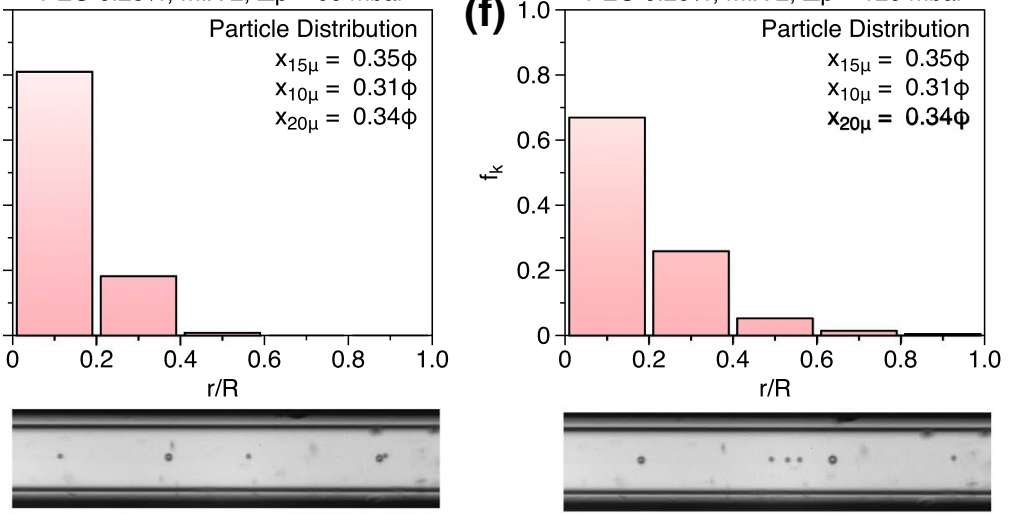

(h) 1.0 PEO $0.25 \%, M I X 2, \Delta p=480 \mathrm{mbar}$

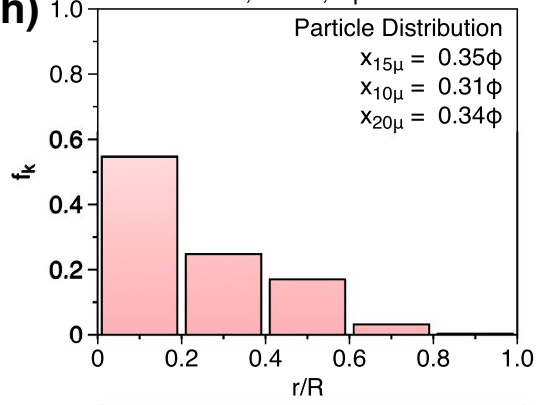

(i)
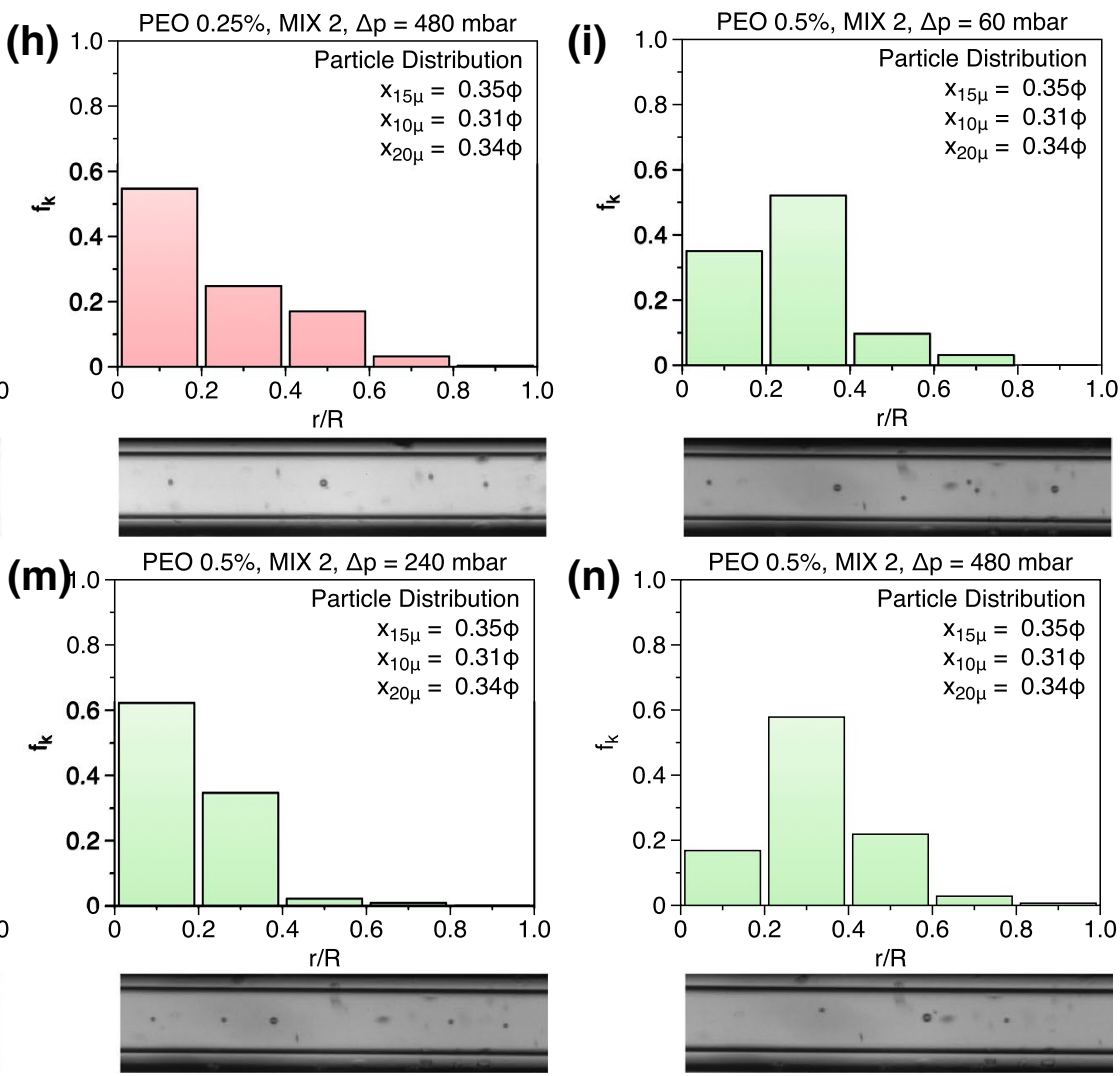

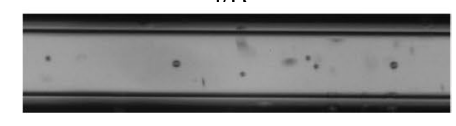

(n) $1.0 \frac{\text { PEO } 0.5 \%, M I X 2, \Delta p=480 \text { mbar }}{\text { Particle Distribution }}$
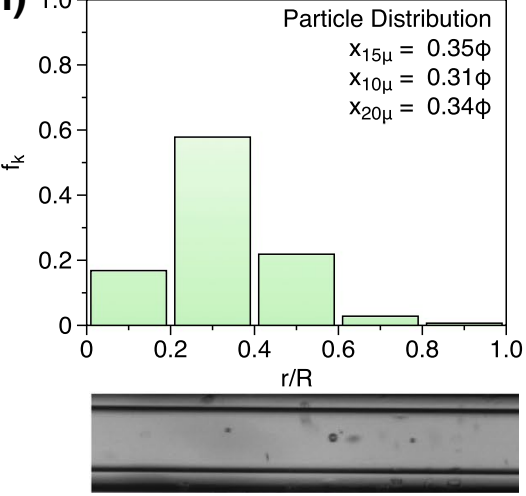
4Fig. 4 Normalised particle fraction $f_{k}$ (Eq. 1) as a function of the normalised radial position for the mixture MIX2. a-d Distribution for the PEO $0.1 \mathrm{wt} \%$ at different pressure drops $\Delta p$ in the range of $30<\Delta p<480$ mbar. Experimental snapshots are also reported below each graph. e-h Distribution for the PEO $0.25 \mathrm{wt} \%$ at different pressure drops $\Delta p$ in the range of $60<\Delta p<480$ mbar. i-n Distribution for the PEO $0.5 \mathrm{wt} \%$ at different pressure drops $\Delta p$ in the range of $60<\Delta p<480$ mbar. Flow is from left to right. Channel internal diameter is $150 \mu \mathrm{m}$. The normalised fraction of particles $f_{k}$ was evaluated through the tracking of at least 100 particles

shear-thinning is detrimental for the overall alignment of a polydisperse system of particles. More specifically, larger $20 \mu \mathrm{m}$ particles (confinement ratio $\beta=0.13$ ) remained substantially on the channel centreline, while smaller $10 \mu \mathrm{m}$ particles (confinement ratio $\beta=0.067$ ) were scattered over the cross-section, in agreement with the experiments in PEO 0.25 wt $\%$ and with the results on particle migration in strongly shear-thinning liquids and square-shaped geometries previously obtained numerically by Villone et al. (2013) and Li et al. (2015), and experimentally by Del Giudice et al. (2017).

So far, only the overall effect of particle polydispersity on the focusing mechanism was presented. It is now interesting to see whether or not the experimentally measured normalised fraction of polydisperse particles aligned on the centreline can be obtained as a combination of the normalised fractions of monodisperse particles aligned on the centreline. Romeo et al. (2013) and Del Giudice et al. (2013) showed that the normalised fraction of particles aligned on the centreline for a monodisperse suspension of non-interacting particles flowing in circular and square-shaped microchannel can be expressed as:

$f_{\text {aligned }}=\frac{1}{1+B e^{-C \Theta^{2}}}$,

where $B=2.7$ and $C=2.75$ are best fit parameters (Del Giudice et al. 2015a), while the parameter $\Theta$ is defined as:

$\Theta=D e \frac{L_{z}}{D} \beta^{2}$.

Equation (9) is valid for vanishing values of $D e$ (shear-thinning is not accounted) and for confinement ratios $\beta \simeq 0.1$. Since $\Theta$ depends on the confinement ratio $\beta$, the fraction $f_{\text {aligned }}$ differs depending on the particle diameter employed. The value $f_{\text {poly }}$ of a polydisperse system (such those measured experimentally) can be estimated as:

$f_{\text {poly }}=\frac{x_{10} f_{\text {aligned,10 }}+x_{15} f_{\text {aligned,15 }}+x_{20} f_{\text {aligned, } 20}}{\phi}$.

By looking at the values of $f_{\text {aligned }}, f_{\text {poly }}$ and $f_{1}$ for MIX1 at $D e<1$ (i.e., in the constant-viscosity region) reported in Table 2, it is possible to see that the values of $f_{\text {poly }}$ are always different from those of $f_{1}$. Such difference is small for the PEO $0.1 \mathrm{wt} \%$ but it is more significant in all the other cases.
Taken together, these results suggest that the experiments with polydisperse systems are not always equivalent to independent experiments with monodisperse particles. This can be due to hydrodynamic interactions between particles with different diameters in confined flows, even though the suspension is considered macroscopically dilute (the volume fraction is just $\phi=0.1 \%$ ). Indeed, when particles align at the centreline, the local volume fraction differs from the macroscopic one, as particles are arranged along the centreline only, and they are not spread over the whole cross-section. In these conditions, particles are likely to interact with their neighbours even at very small macroscopic volume fractions, as demonstrated experimentally by Del Giudice et al. (2018) and numerically by D'Avino and Maffettone (2019).

The viscoelastic focusing of the polydisperse particle mixture MIX2 was also studied in the three fluids under investigation (Fig. 4). Compared to MIX1, the concentration of $10 \mu \mathrm{m}$ particles was larger by $10 \%$, that of $15 \mu \mathrm{m}$ particles was smaller by $20 \%$, and that of $20 \mu \mathrm{m}$ particles was larger by $20 \%$. For the near constant-viscosity PEO $0.1 \mathrm{wt} \%$, the focusing efficiency was lower than that of MIX1, in the whole range of imposed pressure drops. Therefore, the impact of smaller particles on the focusing efficiency in PEO $0.1 \mathrm{wt} \%$ was dominant compared to that of larger particles, as the values of $f_{1}$ for MIX2 decreased on average by $15 \%$ compared to MIX1. For the mixture MIX2 in PEO $0.25 \mathrm{wt} \%$ at $D e<1$, the focusing efficiency dropped on average by around $5 \%$ compared to MIX1, in qualitative agreement with the data in PEO $0.1 \mathrm{wt} \%$. At $D e>1$, instead, particles were found to be more aligned on the centreline compared to MIX1. This observation can be justified from comparison with the experimental results of Del Giudice et al. (2017) and the numerical simulations of Villone et al. (2013). In their work, for confinement ratios $\beta>0.1$ and $D e>1$, particles were mainly focused on the centreline and only very large values of the flow rate could lead to a reduction of the centreline focusing efficiency. This condition finds agreement with the experimental observations presented here, where the larger amount of bigger particles resulted in an increase of the focusing efficiency. For the mixture MIX2 in PEO $0.5 \mathrm{wt} \%$ at $D e \leq 1$, agreement with the results for PEO $0.1 \mathrm{wt} \%$ and for PEO $0.25 \mathrm{wt} \%$ at $D e<1$ was found. At $\Delta p=480 \mathrm{mbar}(D e=6.03)$ the fraction of particles in the region $0.2<r / R<0.4$ was larger compared to that of MIX1, with an overall reduced dispersion compared to MIX1, in agreement with the results for PEO $0.25 \mathrm{wt} \%$ at $D e>1$.

As a final note, the experimental values of $f_{1}$ were different from the theoretical ones $f_{\text {poly }}$ of Eq. (11), analogously to the MIX1 case (Table 2). 


\section{Conclusions}

In this work, the effect of particle polydispersity on the viscoelastic focusing in a straight cylindrical microchannel was studied by preparing two particle suspensions labelled as MIX1 and MIX2, containing different concentrations of particles with diameters of $10 \pm 1 \mu \mathrm{m}, 15 \pm 1.5 \mu \mathrm{m}$ and $20 \pm 2 \mu \mathrm{m}$, suspended in three solutions of polyethylene oxide (PEO) with mass concentrations of $0.1 \mathrm{wt} \%, 0.25 \mathrm{wt} \%$ and $0.5 \mathrm{wt} \%$. When the fluid explored the constant-viscosity region of the rheological curve, up to $95 \%$ of the particles were aligned on the channel centreline. When the fluid explored the shear-thinning region of the rheological curve, centreline focusing efficiency decreased, with the highest value of aligned particles being of $60 \%$. For both mixtures, it was also observed that the fraction of aligned particles in a polydisperse system was not equivalent to that derived from the estimate of independent experiments with monodisperse particles. This phenomenon was ascribed to potential hydrodynamic interactions between particles with different diameters in confined flows, even though the suspension was macroscopically considered dilute.

Acknowledgements The author acknowledges support from EPSRC Platform Grant ref. EP/N013506/1.

Open Access This article is distributed under the terms of the Creative Commons Attribution 4.0 International License (http://creativeco mmons.org/licenses/by/4.0/), which permits unrestricted use, distribution, and reproduction in any medium, provided you give appropriate credit to the original author(s) and the source, provide a link to the Creative Commons license, and indicate if changes were made.

\section{References}

Ahn SW, Lee SS, Lee SJ, Kim JM (2015) Microfluidic particle separator utilizing sheathless elasto-inertial focusing. Chem Eng Sci 126:237-243

Asghari M, Serhatlioglu M, Ortaç B, Solmaz ME, Elbuken C (2017) Sheathless microflow cytometry using viscoelastic fluids. Sci Rep 7(1): 12,342

Bird RB, Carreau PJ (1968) A nonlinear viscoelastic model for polymer solutions and melts-I. Chem Eng Sci 23(5):427-434

Bird RB, Armstrong RC, Hassager O (1987) Dynamics of polymeric liquids. vol. 1: fluid mechanics

Brown M, Wittwer C (2000) Flow cytometry: principles and clinical applications in hematology. Clin Chem 46(8):1221-1229

Cha S, Shin T, Lee SS, Shim W, Lee G, Lee SJ, Kim Y, Kim JM (2012) Cell stretching measurement utilizing viscoelastic particle focusing. Anal Chem 84(23):10,471-10,477

Dannhauser D, Rossi D, Causa F, Memmolo P, Finizio A, Wriedt T, Hellmers J, Eremin Y, Ferraro P, Netti P (2015) Optical signature of erythrocytes by light scattering in microfluidic flows. Lab Chip 15(16):3278-3285

D'Avino G (2013) Non-newtonian deterministic lateral displacement separator: theory and simulations. Rheol Acta 52(3):221-236
D’Avino G, Maffettone PL (2019) Numerical simulations on the dynamics of trains of particles in a viscoelastic fluid flowing in a microchannel. Meccanica

D'Avino G, Romeo G, Villone MM, Greco F, Netti PA, Maffettone PL (2012) Single line particle focusing induced by viscoelasticity of the suspending liquid: theory, experiments and simulations to design a micropipe flow-focuser. Lab Chip 12(9):1638-1645

D'Avino G, Hulsen MA, Maffettone PL (2013) Dynamics of pairs and triplets of particles in a viscoelastic fluid flowing in a cylindrical channel. Comput Fluids 86:45-55

D’Avino G, Greco F, Maffettone PL (2017) Particle migration due to viscoelasticity of the suspending liquid and its relevance in microfluidic devices. Annu Rev Fluid Mech 49:341-360

D’Avino G, Hulsen MA, Greco F, Maffettone PL (2019) Numerical simulations on the dynamics of a spheroid in a viscoelastic liquid in a wide-slit microchannel. J Non-Newton Fluid Mech 263:33-41

De Santo I, D'Avino G, Romeo G, Greco F, Netti PA, Maffettone PL (2014) Microfluidic Lagrangian trap for Brownian particles: three-dimensional focusing down to the nanoscale. Phys Rev Appl 2(6):064,001

Del Giudice F, Romeo G, D’Avino G, Greco F, Netti PA, Maffettone PL (2013) Particle alignment in a viscoelastic liquid flowing in a square-shaped microchannel. Lab Chip 13(21):4263-4271

Del Giudice F, D’Avino G, Greco F, De Santo I, Netti PA, Maffettone PL (2015a) Rheometry-on-a-chip: measuring the relaxation time of a viscoelastic liquid through particle migration in microchannel flows. Lab Chip 15(3):783-792

Del Giudice F, D’Avino G, Greco F, Netti PA, Maffettone PL (2015b) Effect of fluid rheology on particle migration in a square-shaped microchannel. Microfluid Nanofluid 19(1):95-104

Del Giudice F, Sathish S, D’Avino G, Shen AQ (2017) "From the edge to the center": viscoelastic migration of particles and cells in a strongly shear-thinning liquid flowing in a microchannel. Anal Chem 89(24):13,146-13,159

Del Giudice F, D’Avino G, Greco F, Maffettone PL, Shen AQ (2018) Fluid viscoelasticity drives self-assembly of particle trains in a straight microfluidic channel. Phys Rev Appl 10(6):064,058

Erickson D, Li D (2004) Integrated microfluidic devices. Anal Chim Acta 507(1):11-26

Higdon J, Muldowney G (1995) Resistance functions for spherical particles, droplets and bubbles in cylindrical tubes. J Fluid Mech 298:193-210

Ho B, Leal L (1976) Migration of rigid spheres in a two-dimensional unidirectional shear flow of a second-order fluid. J Fluid Mech 76(4):783-799

Holzner G, Stavrakis S, DeMello A (2017) Elasto-inertial focusing of mammalian cells and bacteria using low molecular, low viscosity PEO solutions. Anal Chem 89(21):11,653-11,663

Huang LR, Cox EC, Austin RH, Sturm JC (2004) Continuous particle separation through deterministic lateral displacement. Science 304(5673):987-990

Kang K, Lee SS, Hyun K, Lee SJ, Kim JM (2013) DNA-based highly tunable particle focuser. Nat Commun 4:2567

Kim JA, Lee J, Wu C, Nam S, Di Carlo D, Lee W (2016) Inertial focusing in non-rectangular cross-section microchannels and manipulation of accessible focusing positions. Lab Chip 16(6):992-1001

Kim JY, Ahn SW, Lee SS, Kim JM (2012) Lateral migration and focusing of colloidal particles and dna molecules under viscoelastic flow. Lab Chip 12(16):2807-2814

Lee DJ, Brenner H, Youn JR, Song YS (2013) Multiplex particle focusing via hydrodynamic force in viscoelastic fluids. Sci Rep 3:3258

Leshansky A, Bransky A, Korin N, Dinnar U (2007) Tunable nonlinear viscoelastic "focusing" in a microfluidic device. Phys Rev Lett 98(23):234,501 
Li D, Lu X, Xuan X (2016) Viscoelastic separation of particles by size in straight rectangular microchannels: a parametric study for a refined understanding. Anal Chem 88(24):12,303-12,309

Li G, McKinley GH, Ardekani AM (2015) Dynamics of particle migration in channel flow of viscoelastic fluids. J Fluid Mech 785:486-505

Li Y, Zhang H, Li Y, Li X, Wu J, Qian S, Li F (2018) Dynamic control of particle separation in deterministic lateral displacement separator with viscoelastic fluids. Sci Rep 8(1):3618

Lim EJ, Ober TJ, Edd JF, Desai SP, Neal D, Bong KW, Doyle PS, McKinley GH, Toner M (2014) Inertio-elastic focusing of bioparticles in microchannels at high throughput. Nat Commun 5:4120

Liu C, Xue C, Chen X, Shan L, Tian Y, Hu G (2015) Size-based separation of particles and cells utilizing viscoelastic effects in straight microchannels. Anal Chem 87(12):6041-6048

Liu C, Ding B, Xue C, Tian Y, Hu G, Sun J (2016) Sheathless focusing and separation of diverse nanoparticles in viscoelastic solutions with minimized shear thinning. Anal Chem 88(24):12,547-12,553

Liu C, Guo J, Tian F, Yang N, Yan F, Ding Y, Wei J, Hu G, Nie G, Sun J (2017) Field-free isolation of exosomes from extracellular vesicles by microfluidic viscoelastic flows. ACS Nano 11(7):6968-6976

Lu X, Xuan X (2015) Elasto-inertial pinched flow fractionation for continuous shape-based particle separation. Anal Chem 87(22):11,523-11,530

Lu X, Liu C, Hu G, Xuan X (2017) Particle manipulations in nonnewtonian microfluidics: a review. J Colloid Interface Sci 500:182-201

Nam J, Lim H, Kim D, Jung H, Shin S (2012) Continuous separation of microparticles in a microfluidic channel via the elasto-inertial effect of non-Newtonian fluid. Lab Chip 12(7):1347-1354

Romeo G, D'Avino G, Greco F, Netti PA, Maffettone PL (2013) Viscoelastic flow-focusing in microchannels: scaling properties of the particle radial distributions. Lab Chip 13(14):2802-2807

Schlenk M, Drechsler M, Karg M, Zimmermann W, Trebbin M, Förster S (2018) Splitting and separation of colloidal streams in sinusoidal microchannels. Lab Chip 18(20):3163-3171
Seo KW, Byeon HJ, Huh HK, Lee SJ (2014) Particle migration and single-line particle focusing in microscale pipe flow of viscoelastic fluids. RSC Adv 4(7):3512-3520

Song HY, Lee SH, Salehiyan R, Hyun K (2016) Relationship between particle focusing and dimensionless numbers in elasto-inertial focusing. Rheol Acta 55(11-12):889-900

Stoecklein D, Di Carlo D (2018) Nonlinear microfluidics. Anal Chem 91(1):296-314

Villone M, D'avino G, Hulsen M, Greco F, Maffettone P (2013) Particle motion in square channel flow of a viscoelastic liquid: migration vs. secondary flows. J Non-Newton Fluid Mech 195:1-8

Xiang N, Zhang X, Dai Q, Cheng J, Chen K, Ni Z (2016) Fundamentals of elasto-inertial particle focusing in curved microfluidic channels. Lab Chip 16(14):2626-2635

Yang S, Kim JY, Lee SJ, Lee SS, Kim JM (2011) Sheathless elastoinertial particle focusing and continuous separation in a straight rectangular microchannel. Lab Chip 11(2):266-273

Yin H, Marshall D (2012) Microfluidics for single cell analysis. Curr Opin Biotechnol 23(1):110-119

Yuan D, Zhang J, Yan S, Peng G, Zhao Q, Alici G, Du H, Li W (2016) Investigation of particle lateral migration in sample-sheath flow of viscoelastic fluid and Newtonian fluid. Electrophoresis 37(15-16):2147-2155

Yuan D, Zhao Q, Yan S, Tang SY, Alici G, Zhang J, Li W (2018) Recent progress of particle migration in viscoelastic fluids. Lab Chip 18(4):551-567

Zhang Y, Zhang J, Tang F, Li W, Wang X (2018) Design of a singlelayer microchannel for continuous sheathless single-stream particle inertial focusing. Anal Chem 90(3):1786-1794

Publisher's Note Springer Nature remains neutral with regard to jurisdictional claims in published maps and institutional affiliations. 